\section{Percepção de barreiras e facilitadores dos usuários para participação em programas de promoção da atividade física}

\author{
Perception of barriers and facilitators for users to \\ participate in physical activity programs
}

\section{Percepción de barreras y facilitadores por parte de los usuarios para la participación en programas de promoción de la actividad física}

\section{Resumo}

No Brasil, a atividade física é eixo prioritário das ações de promoção da saúde no Sistema Único de Saúde (SUS). O presente estudo tem como objetivo descrever o perfil dos usuários, as barreiras e os facilitadores para participação em programas para promoção de atividades físicas na atenção básica à saúde. Trata-se de um estudo transversal com abrangência estadual realizado nos municípios de Pernambuco. Foram entrevistados de 2 a 21 individuos, por município, por meio de instrumento previamente testado e validado nas dimensões: sociodemográfica; estado de saúde; participação; informações sobre o programa; motivos de participação; facilitadores para a prática; barreiras para a prática e preferência de atividades. Foram realizadas análises descritivas e inferenciais (qui-quadrado). A amostra foi de 1.153 usuários, sendo 35,9\% com idade entre 41-59 anos; 90,1\% residentes em áreas urbanas; 58,2\% dos usuários participavam das atividades há, pelo menos, um ano. A frequência semanal de 3-4 dias foi de 44,9\%, e 71,1\% praticavam atividades de uma hora ou mais. Observou-se que 40\% das barreiras para a participação nos programas de atividade física e 77,5\% dos facilitadores relatados foram de domínio intrapessoal. A barreira mais prevalente foi "condição atual de saúde", e o facilitador foi "ter uma condição melhor de saúde". As mulheres percebem mais barreiras do que os homens. Conclui-se que as barreiras e os facilitadores de domínio intrapessoal, relacionados com a saúde, são os fatores envolvidos na manutenção e no engajamento dos usuários dos programas e intervenções para promoção da atividade física desenvolvidos pela atenção básica à saúde do Estado de Pernambuco.

Exercício; Promoção da Saúde; Atenção Primária à Saúde
Caroline Ramos de Moura Silva 1

Jorge Bezerra 1

Fernanda Cunha Soares 1

Jorge Mota 2

Mauro Virgílio Gomes de Barros 1

Rafael Miranda Tassitano 1,3

doi: 10.1590/0102-311X00081019

\section{Correspondência}

C. R. M. Silva

Rua Castro Alves 437, Recife, PE 52030-060, Brasil.

1 Grupo de Pesquisa em Estilos de Vida e Saúde, Universidade de Pernambuco, Recife, Brasil.

2 Centro de Investigação em Actividade Física, Saúde e Lazer, Universidade do Porto, Porto, Portugal.

3 Universidade Federal Rural de Pernambuco, Recife, Brasil. 


\section{Introdução}

Programas públicos que ofertam atividade física em nível comunitário são uma das ações fundamentais para a promoção da saúde nos sistemas de saúde de diversos países 1,2. No Brasil, a Política Nacional de Promoção da Saúde (PNPS) tem a atividade física como um eixo prioritário para ampliação e qualificação de ações de promoção da saúde nos serviços ofertados pelo Sistema Único de Saúde (SUS) ${ }^{3}$. Apesar da recente tendência de aumento da prática de atividade física observada nas últimas décadas na população brasileira 4,5, esse não ocorre de forma homogênea entre os diferentes subgrupos populacionais, indivíduos que apresentam menor faixa etária e aqueles com maior escolaridade apresentam maiores níveis de atividade física 4.

Estudos anteriores identificaram que os usuários desses serviços são, principalmente, mulheres, idosos e pessoas acometidas por alguma doença crônica 6,7,8. A baixa participação de alguns subgrupos (homens, crianças e adolescentes) em programas para promoção da atividade física pode estar relacionada às características de funcionamento, como horário e tipo de atividades realizadas nas aulas. O tipo de atividade ofertada é uma das principais variáveis que interfere para o engajamento e a participação do indivíduo na prática de atividade física 9,10 .

Nesse contexto, é importante entender os fatores ambientais, sociais e biológicos que influenciam a participação em programas de atividade física 11,12. Dessa maneira, o planejamento das atividades ofertadas nos programas deve ser levado em conta, além das características peculiares de cada subgrupo 12,13, como, por exemplo, as causas de abandono das aulas por parte dos usuários. Os princípios do SUS de universalidade, equidade e integralidade devem ser aplicados aos programas de promoção de saúde com eixo na prática de atividade física, considerando as singularidades e especificidades dos indivíduos 14 .

Os estudos publicados sobre estrutura e funcionamento de programas para promoção da atividade física são específicos para uma amostra de usuários de um determinado programa e comumente focados em subgrupos específicos como adultos e/ou idosos 15,16,17. A identificação de barreiras e facilitadores para o uso de programas de saúde com ações para promoção da atividade física é uma importante ferramenta que possibilita o replanejamento das ações com o objetivo de aumentar a adesão e manutenção dos usuários nesses serviços 13,18,19. Portanto, o presente estudo tem como objetivo descrever o perfil dos usuários, as barreiras e os facilitadores para participação desses em programas para promoção da atividade física desenvolvidos na atenção básica à saúde do Estado de Pernambuco, Brasil.

\section{Métodos}

Trata-se de um estudo transversal com abrangência estadual, integrante do projeto intitulado $S U S+A-$ tivo - Avaliação dos Programas e Intervenções Relacionados à Atividade Física na Rede de Atenção Básica à Saúde no Estado de Pernambuco, aprovado pelo Comitê de Ética em Pesquisa com Seres Humanos da Universidade de Pernambuco (no 293.601). Informações detalhadas do procedimento metodológico do Projeto SUS+Ativo estão descritas por Barros et al. 20.

Em linhas gerais, foi realizado um mapeamento, junto à Secretaria de Saúde do Estado e às Secretarias Municipais de Saúde, sobre quais e quantos municípios no Estado de Pernambuco tinham o Programa Academia da Saúde (PAS), Programa Academia das Cidades (PACID), Programa Academia da Cidade (PAC), Núcleo de Apoio à Saúde da Família (NASF), Programa Mãe Coruja ou um programa municipal para promoção da atividade física, vinculados às ações da atenção básica à saúde. Foram considerados elegíveis todos os municípios que tinham, pelo menos, um programa ou intervenção para promoção da atividade física citado acima. As informações sobre implementação, tempo de existência e horário de funcionamento dos programas e intervenções foram fornecidas pelos gestores/ coordenadores municipais ou pelos professores durante a coleta de dados.

Considerando as especificidades administrativas de cada município, não foi possível levantar a quantidade de usuários, de atendimentos, de perfil etário e de condição social. Sendo assim, adotou-se uma estratégia específica para determinar a quantidade mínima de usuários a serem entrevistados. Dois critérios foram utilizados para o dimensionamento da amostra: (1) tamanho da população do município (< 20 mil; 20-49,9 mil; 50-99,9 mil e $\geq 100$ mil habitantes) e (2) o tipo e o número de 
programas e intervenções para promoção da atividade física existentes no município (Programa Mãe Coruja; NASF e os programas PAS, PACID e PAC ou similar). A combinação de todas as possibilidades de agrupamento entre o tamanho do município e o tipo de intervenção resultou em sete diferentes cenários amostrais. Esses critérios foram adotados por considerar que o tamanho do município interfere na quantidade de usuários, e que o tipo de programa e intervenção apresenta organizações administrativas e estruturais diferentes (p.ex.: canto, polo, núcleo), além de possuir especificidades no atendimento (p.ex.: o Programa Mãe Coruja atende gestante).

Em cada município, o total necessário de usuários a serem entrevistados variou de 2 (p.ex.: município que ofertava apenas o Programa Mãe Coruja e com população inferior a 20 mil habitantes) a 21 indivíduos (p.ex.: ofertava Programa Mãe Coruja, NASF e o PAS ou PAC e com população superior a 100 mil habitantes). Ao chegar ao local onde era oferecido o programa de atividade física, os pesquisadores escolhiam os usuários para participar da pesquisa, aleatoriamente, até atingir o número estipulado pelo cálculo amostral. Para ser incluído no estudo, era preciso ser usuário do programa de atividade física há, pelo menos, uma semana.

Em cada município, o trabalho de campo foi realizado em uma única visita por uma equipe composta por quatro a cinco pesquisadores, nos horários e locais onde ocorria a oferta da atividade física dos programas e intervenções. Os dados foram coletados em entrevista face a face, por meio da aplicação do instrumento intitulado Questionário para Avaliação de Intervenções para Promoção da Atividade Física na Atenção Básica à Saúde (disponível em: http://www.gpesupe.org/downloads.php), previamente testado e validado 20 . O projeto apresenta quatro instrumentos, sendo um roteiro de observação do ambiente, um questionário para os gestores, um para os profissionais e um para os usuários; neste estudo, foi utilizada apenas a versão do usuário.

Para o presente estudo, foram utilizadas as informações obtidas pelas entrevistas aos usuários acerca das seguintes dimensões: (a) dimensão sociodemográfica: sexo (masculino ou feminino), faixa etária (10-19, 20-40, 41-59 anos e $\geq 60$ anos), trabalho (sim ou não), estado civil (solteiro, casado(a) ou vivendo com parceiro(a), outro) e local de residência (urbana ou rural); (b) dimensão estado de saúde: percepção de saúde (boa ou ruim) e contribuição da participação no programa para melhorar a saúde (sim ou não); (c) participação: tempo de participação no programa/intervenção (<1, 1-11, $\geq 12$ meses), frequência semanal de participação em atividades físicas (1-2, 3-4, $\geq 5$ dias/semana), duração das atividades físicas ( $<30,30-59, \geq 60$ minutos), tempo de deslocamento de casa até o local de atendimento $(<10,10-29, \geq 30$ minutos), meio de transporte que utiliza para se deslocar até o local de atendimento (ativo; passivo), compuseram as informações sobre o programa, principal motivo de participação (identificado dentre 15 opções de resposta), principal facilitador para a prática (identificado dentre 17 opções de resposta), principal barreira para a prática (identificada dentre 16 opções de resposta).

Para essas duas últimas dimensões, foram criadas categorias baseadas nos modelos ecológicos, propostos por Bronfenbrenner ${ }^{21}$ e McLeroy et al. 22 , intrapessoal (conhecimento, atitude, habilidades e a história dos indivíduos), interpessoal (apoio social, da família, dos colegas de trabalho e as redes de amizade do indivíduo) e ambiental (características, organização, estrutura e políticas relacionadas aos programas e intervenções). Os facilitadores foram classificados em: interpessoal (ter um amigo ou uma pessoa da família para fazer companhia; ter menos obrigações familiares; ser incentivado por alguém da minha família; ser incentivado pelo meu médico e ser incentivado por outro profissional de saúde); intrapessoal (trabalhar menos e ter mais tempo livre; ter uma condição de saúde melhor; ter mais disposição, ânimo e energia; ter mais vontade; lidar melhor com timidez; sentir mais confiança nas minhas habilidades para participar das atividades oferecidas; ter mais dinheiro); ambiental (morar mais perto; trabalhar mais perto; sentir que o local onde as atividades são oferecidas é seguro; ter mais e melhores instalações e equipamentos). Do mesmo modo, as barreiras foram classificadas em: interpessoal (falta de amigos ou de companhia; obrigações familiares); intrapessoal (excesso de trabalho; condição atual de saúde; cansaço ou falta de energia; falta de vontade; timidez; falta de habilidade; falta de dinheiro) e ambiental (clima desfavorável; distância do local onde mora; distância do local onde trabalha; falta de segurança no local onde as atividades são oferecidas; instalações e equipamentos inadequados ou inexistentes). Além da opção "não percebo dificuldade".

Por fim, foi perguntado quais atividades do programa/intervenção eram oferecidas à população e quais que gostariam que fossem ofertadas. Foi apresentada uma lista com 12 atividades (grupos de caminhada, dança, práticas integrativas, ginástica, exercícios de alongamento/relaxamento, exercícios 
de força/resistência muscular, jogos e brincadeiras, passeios, lutas/artes marciais, esporte, avaliação física e educação em saúde) na qual o(a) usuário(a) poderia responder mais de uma opção.

A tabulação dos dados foi realizada por meio da leitura óptica dos questionários, mediante utilização do software SPHYNX (https://www.sphynxsoftware.com), e os dados foram analisados no programa SPSS (https://www.ibm.com/). As análises dos dados incluíram procedimentos de estatística descritiva, tais como distribuição de frequências (relativa e absoluta), e, para análise inferencial, foi utilizado o teste do qui-quadrado, sendo adotado um valor de $\mathrm{p}<0,05$ para nível de significância.

\section{Resultados}

A amostra final do estudo foi de 1.153 usuários, e informações demográficas, sociais, do programa e relacionadas à saúde estão apresentadas na Tabela 1. Em relação às características demográficas, 35,9\% dos usuários tinham entre 41-59 anos, 90,1\% residiam em áreas urbanas, 52,4\% eram casados ou viviam com companheiro, e $66,4 \%$ trabalhavam. No que se refere ao tempo de participação, $58,2 \%$ dos usuários participavam das atividades há, pelo menos, um ano, 44,9\% referiram ter uma frequência semanal de 3-4 dias, 71,1\% informaram que a duração das atividades era de uma hora ou mais. A percepção de saúde foi considerada boa por 60,3\% dos participantes, e 99,2\% consideram que as atividades realizadas nos programas e intervenções contribuem para a saúde (Tabela 1).

Dentre os principais motivos que levam os usuários a participarem das atividades que são oferecidas pelos programas/intervenções, 53,6\% relataram "melhorar a saúde”, 6,7\% "prevenir doenças", $6,6 \%$ emagrecer, e 33,1\% relataram outros motivos. As atividades ofertadas à população mais relatadas pelos usuários foram alongamento e relaxamento (92,8\%), ginástica $(81,8 \%)$ e exercícios de força e resistência muscular (70,4\%) (Figura 1). Dentre as atividades que gostariam que fossem ofertadas, as mais frequentes foram esportes (49\%), práticas integrativas (48,3\%) e passeios (48\%), conforme apresentado na Figura 1.

Na Tabela 2, observa-se que 44\% das barreiras e 77,5\% dos facilitadores relatados foram de domínio intrapessoal. Para os homens, $46,8 \%$ das barreiras e $71 \%$ dos facilitadores eram intrapessoais, da mesma forma, para as mulheres, $43,8 \%$ das barreiras e $78,1 \%$ dos facilitadores foram de domínio intrapessoal. As barreiras interpessoais foram citadas por 20,3\% das mulheres e 9,6\% dos homens. Com relação às barreiras de domínio intrapessoal, 37,8\% dos usuários tinham idade entre 10-19 anos; 43,8\%, entre 20-40 anos; e 46,2\% eram $\geq 60$ anos de idade. Os facilitadores de domínio intrapessoal foram relatados por $67,9 \%$ dos usuários entre 10-19 anos, 74,4\% entre os de $20-40$ anos e 77,9\% entre os que apresentavam $\geq 60$ anos de idade (Tabela 3).

Adicionalmente a esses resultados, a "condição atual de saúde" foi relatada como principal barreira percebida por $23,1 \%$ das mulheres e $19,2 \%$ dos homens. Os homens que disseram "não perceber dificuldade" foram 19,2\%. Como agente facilitador, "ter uma condição melhor de saúde" foi descrita por $51 \%$ dos homens e $53,9 \%$ das mulheres.

Com relação à faixa etária, 19,9\% dos usuários entre 20-59 anos e 30,1\% com idade $\geq 60$ anos relataram "a condição atual de saúde" como principal barreira percebida, porém a maioria dos usuários, entre 10-19 anos (20,7\%), respondeu não perceber nenhuma barreira. Para os facilitadores, "ter uma condição de saúde melhor" foi o mais referido entre os usuários de todas as faixas etárias, entre 10-19 anos (42\%), entre 20-40 anos (51,2\%), entre 41-59 anos (63,7\%) e entre os com idade $\geq 60$ anos $(62 \%)$.

A percepção de barreiras foi estratificada pelo tempo de participação dos usuários nos programas e intervenções. Do total de usuários, $81,1 \%(\mathrm{n}=843)$ perceberam alguma barreira.

Os usuários que perceberam algum tipo de barreira, 41,8\% participavam do programa há menos de um ano. Já os usuários que não perceberam nenhuma barreira, 31\% participavam do programa há mais de dois anos (Figura 2). 


\section{Tabela 1}

Características demográficas e da participação dos usuários nos programas/intervenções de promoção da atividade física de acordo com o sexo. Pernambuco, Brasil, 2014-2016.

\begin{tabular}{|c|c|c|c|c|c|c|c|}
\hline \multirow[t]{2}{*}{ Variáveis } & \multicolumn{2}{|c|}{ Total } & \multicolumn{2}{|c|}{ Masculino } & \multicolumn{2}{|c|}{ Feminino } & \multirow[t]{2}{*}{ Valor de $\mathrm{p}$} \\
\hline & $\mathbf{n}$ & $\%$ & $\mathrm{n}$ & $\%$ & $\mathrm{n}$ & $\%$ & \\
\hline \multicolumn{8}{|l|}{ Faixa etária (anos) } \\
\hline $10-19$ & 87 & 7,5 & 28 & 26,4 & 59 & 5,6 & \multirow{4}{*}{0,001} \\
\hline $20-40$ & 313 & 27,1 & 19 & 17,9 & 294 & 28,1 & \\
\hline $41-59$ & 414 & 35,9 & 22 & 20,7 & 392 & 37,4 & \\
\hline$\geq 60$ & 339 & 29,4 & 37 & 34,9 & 302 & 28,8 & \\
\hline \multicolumn{8}{|l|}{ Local de residência } \\
\hline Urbano & 1.032 & 90,1 & 89 & 84,8 & 943 & 90,6 & \multirow{2}{*}{0,060} \\
\hline Rural & 114 & 9,9 & 16 & 15,2 & 98 & 9,4 & \\
\hline \multicolumn{8}{|l|}{ Estado civil } \\
\hline Solteiro & 300 & 26,1 & 49 & 46,2 & 251 & 24,1 & \multirow{3}{*}{0,001} \\
\hline Casado/Vivendo com parceiro & 602 & 52,4 & 48 & 45,3 & 554 & 53,2 & \\
\hline Outro & 246 & 21,4 & 9 & 8,5 & 237 & 22,7 & \\
\hline \multicolumn{8}{|l|}{ Trabalho } \\
\hline Sim & 762 & 66,4 & 70 & 66,0 & 692 & 66,5 & \multirow[t]{2}{*}{0,920} \\
\hline Não & 385 & 33,6 & 36 & 34,0 & 349 & 33,5 & \\
\hline \multicolumn{8}{|l|}{ Tempo de participação (meses) } \\
\hline$<1$ & 98 & 8,5 & 6 & 5,7 & 92 & 8,8 & \multirow{3}{*}{0,08} \\
\hline $1-11$ & 381 & 33,2 & 45 & 42,5 & 336 & 32,3 & \\
\hline$\geq 12$ & 668 & 58,2 & 55 & 51,9 & 613 & 58,9 & \\
\hline \multicolumn{8}{|l|}{ Frequência semanal (dias) } \\
\hline$\leq 2$ & 412 & 36,8 & 53 & 51,5 & 359 & 35,3 & \multirow{3}{*}{0,005} \\
\hline $3-4$ & 503 & 44,9 & 36 & 35,0 & 467 & 45,9 & \\
\hline$\geq 5$ & 206 & 18,4 & 14 & 13,6 & 192 & 18,9 & \\
\hline \multicolumn{8}{|l|}{ Duração das sessões (minutos) } \\
\hline$<30$ & 55 & 4,8 & 7 & 6,7 & 48 & 4,7 & \multirow{3}{*}{0,600} \\
\hline $30-59$ & 273 & 24,1 & 23 & 21,9 & 250 & 24,3 & \\
\hline$\geq 60$ & 807 & 71,1 & 75 & 71,4 & 732 & 71,1 & \\
\hline \multicolumn{8}{|l|}{ Meio de transporte } \\
\hline Ativo & 1.038 & 90,3 & 77 & 73,3 & 961 & 92,0 & \multirow{2}{*}{0,001} \\
\hline Passivo & 112 & 9,7 & 28 & 26,7 & 84 & 8,0 & \\
\hline \multicolumn{8}{|l|}{ Tempo de deslocamento (minutos) } \\
\hline$<10$ & 600 & 52,3 & 59 & 57,3 & 541 & 51,8 & \multirow{3}{*}{0,550} \\
\hline $10-20$ & 467 & 40,7 & 38 & 36,9 & 429 & 41,1 & \\
\hline$\geq 30$ & 81 & 7,1 & 6 & 5,8 & 75 & 7,2 & \\
\hline \multicolumn{8}{|l|}{ Percepção de saúde } \\
\hline Boa & 695 & 60,3 & 75 & 70,8 & 620 & 59,2 & חבחم \\
\hline Ruim & 458 & 39,7 & 31 & 29,2 & 427 & 40,8 & $0,0<0$ \\
\hline Programa contribui para saúde & & & & & & & \\
\hline Sim & 1.137 & 99,2 & 105 & 99,1 & 1.032 & 99,2 & 0840 \\
\hline Não & 9 & 0,8 & 1 & 0,9 & 8 & 0,8 & 0,040 \\
\hline
\end{tabular}




\section{Figura 1}

Atividades ofertadas e atividades que os usuários gostariam que fossem ofertadas pelos programas e intervenções de atividade física. Pernambuco, Brasil.

1a) Oferta

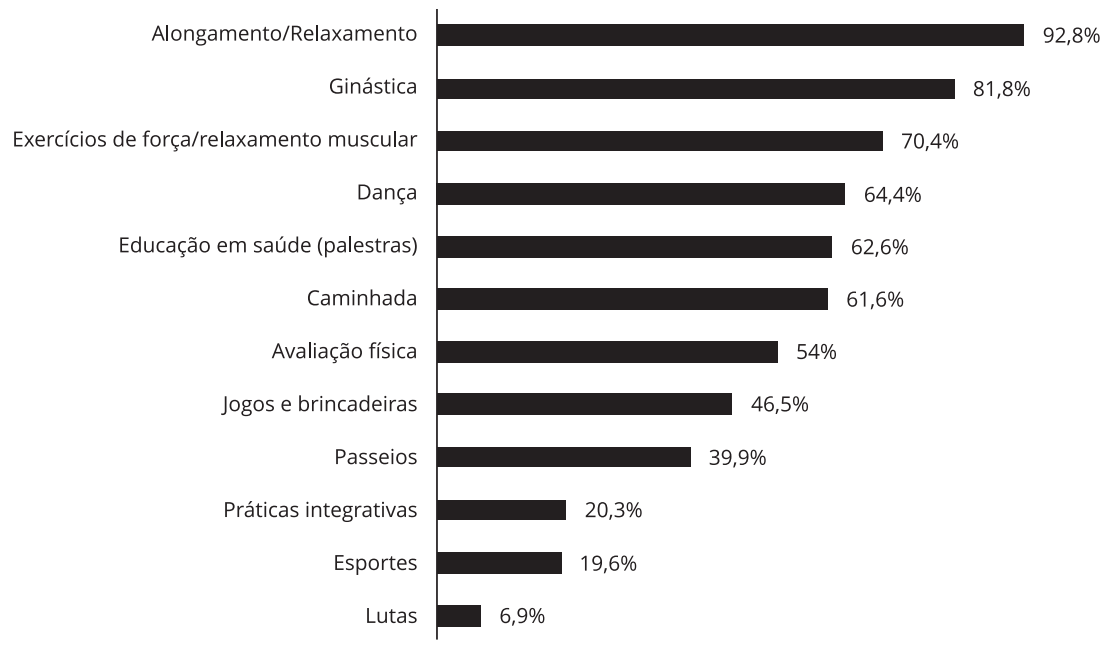

1b) Gostaria que ofertasse

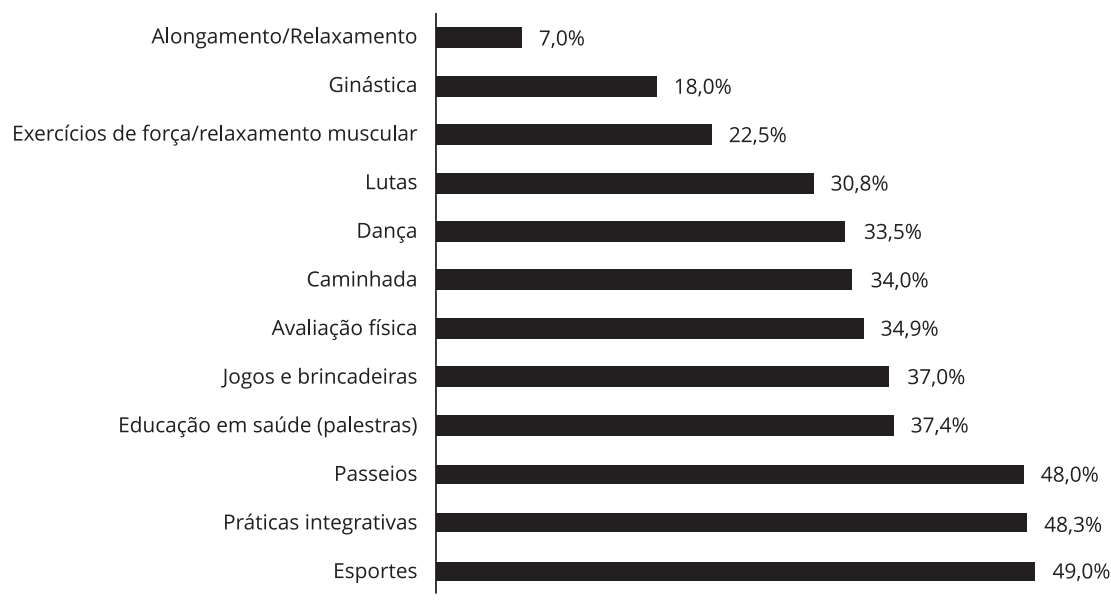




\section{Tabela 2}

Barreiras percebidas e facilitadores para a participação de usuários nos programas e intervenções de atividade física, estratificada por sexo. Pernambuco, Brasil.

\begin{tabular}{|c|c|c|c|c|c|c|c|}
\hline & \multicolumn{2}{|c|}{ Total } & \multicolumn{2}{|c|}{ Feminino } & \multicolumn{2}{|c|}{ Masculino } & \multirow[t]{2}{*}{ Valor de $\mathrm{p}$} \\
\hline & $\mathbf{n}$ & $\%$ & $\mathbf{n}$ & $\%$ & $\mathbf{n}$ & $\%$ & \\
\hline Barreiras percebidas & & & & & & & 0,078 \\
\hline Intrapessoal & 458 & 44,0 & 414 & 43,8 & 44 & 46,8 & \\
\hline Interpessoal & 201 & 19,3 & 192 & 20,3 & 9 & 9,6 & \\
\hline Ambiental & 184 & 16,2 & 163 & 17,2 & 21 & 22,3 & \\
\hline Não percebe dificuldades para participar & 197 & 18,9 & 177 & 18,7 & 20 & 21,3 & \\
\hline Facilitadores para participação & & & & & & & 0,269 \\
\hline Intrapessoal & 809 & 77,5 & 743 & 78,1 & 66 & 71,0 & \\
\hline Interpessoal & 150 & 14,4 & 132 & 13,9 & 18 & 19,3 & \\
\hline Ambiental & 85 & 8,1 & 76 & 7,0 & 9 & 9,7 & \\
\hline
\end{tabular}

\section{Tabela 3}

Barreiras percebidas e facilitadores para a participação de usuários nos programas e intervenções de atividade física, estratificada por faixa etária. Pernambuco, Brasil, 2014-2016.

\begin{tabular}{|c|c|c|c|c|c|c|c|c|c|}
\hline & \multicolumn{8}{|c|}{ Faixa etária (anos) } & \multirow[t]{3}{*}{ Valor de $p$} \\
\hline & \multicolumn{2}{|c|}{$10-19$} & \multicolumn{2}{|c|}{$20-40$} & \multicolumn{2}{|c|}{ 41-59 } & \multicolumn{2}{|c|}{$\geq 60$} & \\
\hline & $\mathbf{n}$ & $\%$ & $\mathbf{n}$ & $\%$ & $\mathbf{n}$ & $\%$ & $\mathbf{n}$ & $\%$ & \\
\hline \multicolumn{10}{|l|}{ Barreiras percebidas } \\
\hline Intrapessoal & 31 & 37,8 & 131 & 46,3 & 155 & 41,9 & 141 & 46,2 & 0,193 \\
\hline Interpessoal & 21 & 25,6 & 63 & 22,3 & 68 & 18,4 & 49 & 16,1 & \\
\hline Ambiental & 12 & 14,6 & 44 & 15,5 & 77 & 20,8 & 51 & 16,7 & \\
\hline Não percebe dificuldades para participar & 18 & 21,9 & 45 & 15,9 & 70 & 18,9 & 64 & 20,1 & \\
\hline \multicolumn{10}{|l|}{ Facilitadores para participação } \\
\hline Intrapessoal & 55 & 67,9 & 209 & 74,4 & 302 & 81,6 & 243 & 77,9 & 0,042 \\
\hline Interpessoal & 19 & 23,5 & 44 & 15,7 & 39 & 10,5 & 48 & 15,4 & \\
\hline Ambiental & 7 & 8,6 & 28 & 9,9 & 29 & 7,8 & 21 & 6,7 & \\
\hline
\end{tabular}


Figura 2

Percepção de barreiras de acordo com o tempo de participação dos usuários nos programas e intervenções de atividade física. Pernambuco, Brasil, 2014-2016.

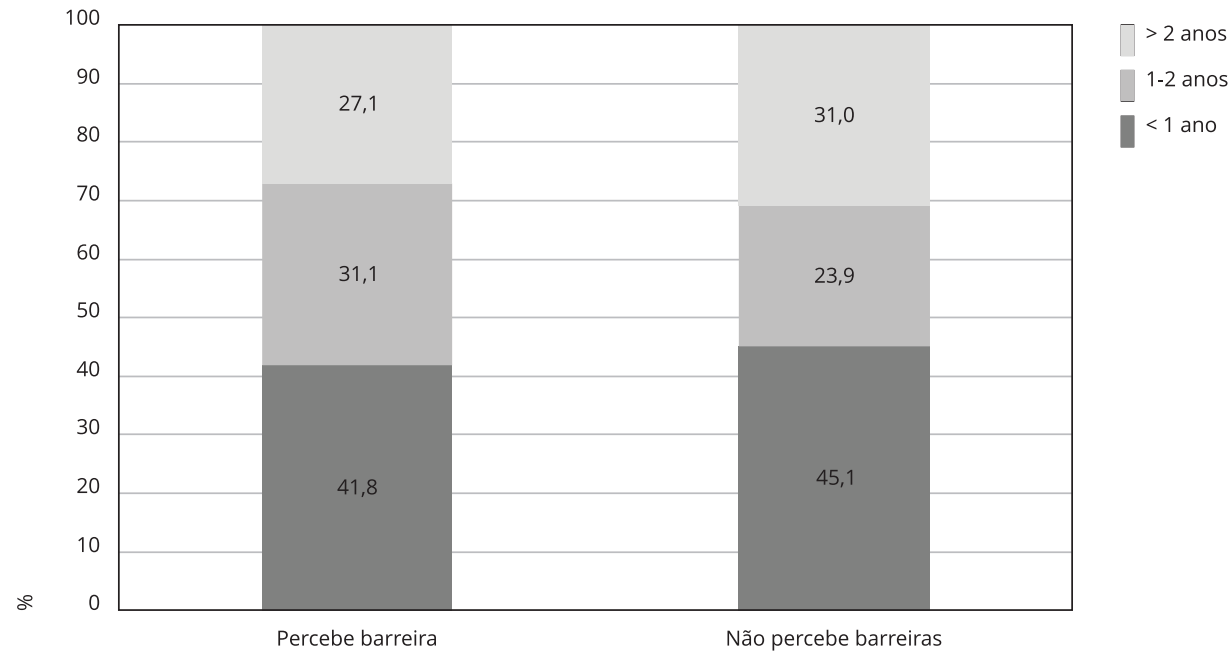

\section{Discussão}

O presente estudo descreveu o perfil de participação, as principais barreiras e os facilitadores percebidos por usuários participantes dos programas e intervenções para promoção da atividade física de um estado brasileiro. A maioria dos usuários atendidos era do sexo feminino e tinha entre 20-59 anos, embora quatro em cada dez usuários referiram percepção negativa da saúde, eles acreditam que a participação nos programas e intervenções contribuiu para a condição de saúde, revelando também que apresentam uma boa frequência e tempo de participação. As barreiras e os facilitadores mais apontados para a participação foram os de domínio intrapessoal, o principal facilitador foi ter uma condição de saúde melhor, e a principal barreira foi a condição atual de saúde, apesar de a maioria relatar ter uma boa percepção de saúde.

A maior participação das mulheres nos programas e intervenções era esperada, uma vez que levantamentos nacionais relacionados a programas relatam que existe essa disparidade entre homens e mulheres participantes. Os homens apresentam, majoritariamente, os maiores níveis de atividade física no lazer e no deslocamento ativo, e essa condição tende a diminuir com o aumento da idade 23 . Mas, quando se observa o cenário das atividades ofertadas, por meio das políticas públicas de promoção da atividade física, a participação das mulheres é maior do que a dos homens 13,24. Esse fato pode ser compreendido pelo tipo de atividade ofertada parecer atrair mais o público feminino.

Segundo a Pesquisa Nacional de Saúde, publicada em 2013 (PNS 2013), as mulheres utilizam mais serviços médicos e têm hábitos mais saudáveis quando comparadas aos homens 25 . Além de que, a maior quantidade de ações desenvolvidas pelo Programa Saúde da Família (PSF) é voltada ao público feminino, incluindo serviços de prevenção ao câncer de colo uterino, pré-natal e planejamento familiar 26, em detrimento de atividades direcionadas ao atendimento, em especial, de homens 27 e adolescentes 28. Cenário que pode contribuir para o percentual elevado de mulheres atendidas na atenção básica e para a maior participação nas ações de promoção da atividade física.

No que se refere à faixa etária, os programas e as intervenções são voltados principalmente para a população idosa, seguidos pelos que atendem ao público adulto ${ }^{6}$. Apesar da expansão dos programas de promoção da atividade física na atenção básica, alguns subgrupos populacionais apresentam baixa participação, como é o caso das crianças e dos adolescentes 6,7. Segundo Garcia \& Fisberg 29, as 
atividades de preferência nesse subgrupo são jogos, lutas, brincadeiras e danças, porém são as menos ofertadas nos programas, resultando na baixa participação ${ }^{6}$. Além disso, os programas, tais como PAC e PACID e as intervenções costumam ocorrer nos horários que o acesso de uma parcela da população fica prejudica por estar estudando ou trabalhando nesse horário.

Este estudo identificou elevada prevalência da barreira "condição atual de saúde" e do facilitador "ter uma condição melhor de saúde", para ambos os sexos e todas as faixas etárias contempladas na amostra. As condições específicas de saúde são encontradas em outros estudos com idosos como sendo a barreira frequentemente associada à prática de atividade física 16,30,31,32,33 .

O fator relacionado à saúde é também apontado na literatura como sendo uma das principais razões para idosos e adultos iniciarem nos programas, principalmente entre os que acreditam nos benefícios da prática da atividade física 12,34,35. Por outro lado, Cardoso et al. 30 apresentam os relacionamentos, "convite de amigos e familiares", como motivo que leva à participação e ao engajamento dos idosos na prática da atividade física. Segundo Freitas et al. 15, a influência e a companhia de amigos e familiares foram os motivos apontados como "sem importância", já os aspectos relacionados à melhora da saúde se destacaram como causas "importantíssimas” pelos idosos. Além disso, outros aspectos identificados na literatura são considerados para a participação e a adesão a um programa de atividades, como o acesso e a localização, o tipo de atividade realizado e o custo ${ }^{36}$.

Tanto entre os idosos 31 quanto entre os adolescentes 37 , o sexo feminino percebe mais barreiras do que o sexo masculino. Em um estudo com escolares da rede pública de Curitiba, Paraná, Brasil, "não ter companhia de amigos", "ter preguiça” e "clima" são as barreiras mais relatadas por adolescentes ativos 37 . Do mesmo modo, "a falta de companhia" foi a barreira com elevada prevalência entre escolares de Londrina, Paraná 38 . Percebe-se que existe uma diversidade de fatores, incluindo os domínios intrapessoal, interpessoal e ambiental que influenciam para adoção e manutenção da prática de atividade física em todas as faixas etárias, incluindo os idosos 39. O tempo de participação parece diminuir a percepção de barreiras. No presente estudo, os usuários que participam há mais de dois anos são os que menos percebem barreiras. Em adição, os usuários que participam há menos de um ano são os que mais as percebem.

Algumas limitações devem ser consideradas, por ser um estudo exploratório, é preciso cautela na interpretação dos resultados. Apesar de todos os programas e intervenções de municípios pernambucanos serem visitados, os resultados devem ser interpretados com cautela, uma vez que a amostra foi escolhida por conveniência. O uso do questionário pode limitar a obtenção de dados devido à omissão de informações por esquecimento ou autocensura por parte dos participantes. Entretanto, o estudo apresenta pontos fortes, em especial a abrangência estadual do Projeto SUS+Ativo, sendo a amostra composta por participantes de diversas cidades do Estado de Pernambuco. Além disso, a coleta realizada in loco permitiu a aproximação com diferentes realidades locais.

Os achados do presente estudo permitem concluir que as barreiras e os facilitares de domínio intrapessoal, relacionados com a saúde, são os fatores envolvidos na manutenção e no engajamento dos usuários dos programas e intervenções para promoção da atividade física desenvolvidos pela atenção básica à saúde do Estado de Pernambuco. A identificação desses fatores pode direcionar os profissionais e os gestores a desenvolverem estratégias de intervenção direcionadas que fortaleçam os facilitadores e, por outro lado, diminuam as barreiras existentes. O aprofundamento das análises permitirá a identificação de fatores associados às barreiras e aos facilitadores contribuindo para atuação, ampliação e qualificação da oferta desses serviços na atenção básica à saúde. 


\section{Colaboradores}

C. R. M. Silva participou na fase de concepção, análise dos dados, redação e versão final do artigo. J. Bezerra, F. C. Soares, J. Mota e M. V. G. Barros colaboraram na revisão das versões anteriores do artigo e aprovação da versão final. R. M. Tassitano supervisionou todas as etapas do estudo e participou na fase de análise dos dados e revisão crítica do estudo.

\section{Informações adicionais}

ORCID: Caroline Ramos de Moura Silva (00000002-8727-0024); Jorge Bezerra (0000-0002-99354508); Fernanda Cunha Soares (0000-0001-64653164); Jorge Mota (0000-0001-7571-9181); Mauro Virgílio Gomes de Barros (0000-0003-3165-0965); Rafael Miranda Tassitano (0000-0002-2713-8670).

\section{Agradecimentos}

Aos pesquisadores e colaboradores do Grupo de Pesquisa em Estilos de Vida e Saúde. O estudo teve o suporte financeiro das agências de financiamento Fundação de Amparo à Ciência e Tecnologia de Pernambuco (FACEPE; no APQ-0287-4.09/13) e Conselho Nacional de Desenvolvimento Científico e Tecnológico (CNPq; no APQ 485911/2013-2). J. Mota teve suporte FCT: SFRH/BSAB/142983/2018 e UID/DTP/00617/2019, bem como do Programa de Bolsas Santander Universidades 2018.

\section{Referências}

1. Waxman A; World Health Assembly. WHO global strategy on diet, physical activity and health. Food Nutr Bull 2004; 25:292-302.

2. Barreto SM, Pinheiro ARO, Sichieri R, Monteiro CA, Batista Filho M, Schmidt MI, et al Análise da Estratégia Global para Alimentação, Atividade Física e Saúde, da Organização Mundial da Saúde. Epidemiol Serv Saúde 2005; 14:41-68.

3. Ministério da Saúde. Política Nacional de Promoção da Saúde: PNPS. Anexo I da Portaria de Consolidação no 2, de 28 de setembro de 2017 , que consolida as normas sobre as políticas nacionais de saúde do SUS. Brasília: Ministério da Saúde; 2018.

4. Cruz MS, Bernal RTI, Claro RM. Tendência da prática de atividade física no lazer entre adultos no Brasil (2006-2016). Cad Saúde Pública 2018; 34:e0014817.

5. Rech CR, Reis RS, Hino AAF, Sá GBAR, Stopa SR, Malta DC. Tendências temporais de indicadores de atividade física e comportamento sedentário nas capitais da Região Sul do Brasil: 2006-2013. Rev Bras Ativ Fís Saúde 2015; 20:47-56.

6. Amorim TC, Knuth AG, Cruz DKA, Malta DC, Reis RS, Hallal PC. Descrição dos programas municipais de promoção da atividade física financiados pelo Ministério da Saúde. Rev Bras Ativ Fís Saúde 2013; 18:63-74.

7. Knuth AG, Malta DC, Cruz DKA, Freitas PC, Lopes MP, Fagundes J, et al. Rede Nacional de Atividade Física do Ministério da Saúde: resultados e estratégias avaliativas. Rev Bras Ativ Fís Saúde 2010; 15:229-33.

8. Costa BV, Bottcher LB, Kokubun E. Aderência a um programa de atividade física e fatores associados. Motriz (Online) 2009; 15:25-36.

9. Dantas EHM. Fatores afetivos indispensáveis para o sucesso nos programas de atividade física para a 3a idade. Rev Bras Ativ Fís Saúde 1997; 2:75-82.

10. Ferreira RW, Caputo EL, Hafele CA, Jerônimo JS, Florindo AA, Knuth AG, et al. Acesso aos programas públicos de atividade física no Brasil: Pesquisa Nacional de Saúde, 2013. Cad Saúde Pública 2019; 35:e00008618.

11. Bauman AE, Reis RS, Sallis JF, Wells JC, Loos RJF, Martin BW, et al. Correlates of physical activity: Why are some people physically active and others not? Lancet 2012; 380:258-71.

12. Andreotti MC, Okuma SS. Perfil sociodemográfico e de adesão inicial de idosos ingressantes em um programa de educação física. Rev Paul Educ Fís 2003; 17:142-53.

13. Hallal PC, Tenório MCM, Tassitano RM, Reis RS, Carvalho YM, Cruz DKA, et al. Avaliação do programa de promoção da atividade física Academia da Cidade de Recife, Pernambuco, Brasil: percepções de usuários e não-usuários. Cad Saúde Pública 2010; 26:70-8. 
14. Secretaria de Atenção à Saúde, Secretaria de Vigilância em Saúde, Ministério da Saúde. Política Nacional de Promoção da Saúde. Revisão da Portaria MS/GM no 687, de 30 de março de 2006. Brasília: Ministério da Saúde; 2015.

15. Freitas CMSM, Santiago MS, Viana A, Leão AC, Freyre C. Aspectos motivacionais que influenciam a adesão e manutenção de idosos a programas de exercícios físicos. Rev Bras Cineantropom Desempenho Hum 2007; 9:92-100.

16. Nascimento CMC, Gobbi S, Hirayama MS, Brazão MC. Nível de atividade física e as principais barreiras percebidas por idosos de Rio Claro. Rev Educ Fís 2008; 19:109-18.

17. Rocha SV, Carneiro LRV, Virtuoso Júnior JS. Exercício físico e saúde em pessoas idosas: qual a relação? Revista Saúde.com 2006; 2:85-90.

18. Hoehner CM, Soares J, Parra-Pérez D, Ribeiro IC, Joshu CE, Pratt M, et al. Physical activity interventions in Latin America: a systematic review. Am J Prev Med 2008; 34:224-33.

19. Martin KA, Sinden AR. Who will stay and who will go? A review of older adults' adherence to randomized controlled trials of exercise. J Aging Phys Act 2001; 9:91-114.

20. Barros M, Lemos E, Silva J, Silva C, Fosenca S, Tassitano R. Evaluation of programs and interventions for physical activity promotion in primary health care in Pernambuco: construction and validation of instruments and fieldwork methods of the SUS+Ativo Project. Rev Bras Ativ Fís Saúde 2016; 21:388-99.

21. Bronfenbrenner U. Toward and experimental ecology of human development. Am Psychol 1977; 32:513-31.

22. McLeroy K, Bibeau D, Steckler A, Glanz K. An ecology perspective on health promotion programs. Health Educ Q 1988; 15:351-77.

23. Departamento de Vigilância de Doenças e Agravos não Transmissíveis e Promoção da Saúde, Secretaria de Vigilância em Saúde, Ministério da Saúde. Vigitel Brasil 2017: vigilância de fatores de risco e proteção para doenças crônicas por inquérito telefônico: estimativas sobre frequência e distribuição sociodemográfica de fatores de risco e proteção para doenças crônicas nas capitais dos 26 estados brasileiros e no Distrito Federal em 2017. Brasília: Ministério da Saúde; 2017.

24. Simões EJ, Hallal P, Pratt M, Ramos L, Munk M, Damascena W, et al. Effects of a community-based, professionally supervised intervention on physical activity levels among residents of Recife, Brazil. Am J Public Health 2009; 99:68-75.

25. Ministério da Saúde; Instituto Brasileiro de Geografia e Estatística. Pesquisa Nacional de Saúde: percepção do estado de saúde, estilos de vida e doenças crônicas. Rio de Janeiro: Instituto Brasileiro de Geografia e Estatística; 2014.
26. Facchini LA, Piccini RX, Tomasi E, Thumé E, Silveira DS, Siqueira FV, et al. Desempenho do PSF no Sul e no Nordeste do Brasil: avaliação institucional e epidemiológica da Atenção Básica à Saúde. Ciênc Saúde Colet 2006; 11:669-81.

27. Figueiredo W. Assistência à saúde dos homens: um desafio para os serviços de atenção primária. Ciênc Saúde Colet 2005; 10:1059.

28. Vieira RP, Gomes SHP, Machado MDFAS, Bezerra IMP, Machado CA. Participation of adolescents in the Family Health Strategy from the theoretical-methodological structure of an enabler to participation. Rev Latinoam Enferm 2014; 22:309-16.

29. Garcia L, Fisberg M. Atividades físicas e barreiras referidas por adolescentes atendidos num serviço de saúde. Rev Bras Cineantropom Desempenho Hum 2011; 13:163-9.

30. Cardoso AS, Borges LJ, Mazo GZ, Benedetti TB, Kuhnen AP. Fatores influentes na desistência de idosos em um programa de exercício físico. Movimento (Porto Alegre) 2008; 14:225-39.

31. Satariano WA, Haight TJ, Tager IB. Reasons given by older people for limitation or avoidance of leisure time physical activity. J Am Geriatr Soc 2000; 48:505-12.

32. Schutzer KA, Graves BS. Barriers and motivations to exercise in older adults. Prev Med 2004; 39:1056-61.

33. Dergance JM, Calmbach WL, Dhanda R, Miles TP, Hazuda HP, Mouton CP. Barriers to and benefits of leisure time physical activity in the elderly: differences across cultures. J Am Geriatr Soc 2003; 51:863-8.

34. Booth ML, Bauman A, Owen N. Perceived barriers to physical activity among older Australians. J Aging Phys Act 2002; 10:271-80.

35. Coghill N, Cooper AR. Motivators and demotivators for adherence to a program of sustained walking. Prev Med 2009; 49:24-7.

36. Cohen-Mansfield J, Marx MS, Biddison JR, Guralnik JM. Socio-environmental exercise preferences among older adults. Prev Med 2004; 38:804-11.

37. Santos MS, Akira A, Hino F, Reis RS, Rodriguez-Añez CR. Prevalência de barreiras para a prática de atividade física em adolescentes. Rev Bras Epidemiol 2010; 13:94-104.

38. Dias DF, Loch MR, Ronque ERV. Barreiras percebidas à prática de atividades físicas no lazer e fatores associados em adolescentes. Ciênc Saúde Colet 2015; 20:3339-50.

39. Baert V, Gorus E, Mets T, Geerts C, Bautmans I. Motivators and barriers for physical activity in the oldest old: a systematic review. Ageing Res Rev 2011; 10:464-74. 


\section{Abstract}

In Brazil, physical activity is a priority area in health promotion in the Brazilian Unified $\mathrm{Na}$ tional Health System (SUS). The study aims to describe users' profiles and the barriers and facilitators in basic healthcare for participation in programs to promote physical activity. This was a statewide cross-sectional study in municipalities (counties) in Pernambuco State. Between 2 and 21 individuals were interviewed per municipality, using a previous tested instrument, validated in the following dimensions: sociodemographic; health status; participation; information on the program; reasons for participation; facilitators; barriers; and preference of activities. Descriptive and inferential analyses (chi-square) were performed. The sample consisted of 1,153 users, $35.9 \%$ of whom between 41-59 years of age; $90.1 \%$ lived in urban areas; $58.2 \%$ had participated in physical activities for at least a year. Weekly frequency of 3-4 days was $44.9 \%$, and $71.1 \%$ practiced activities of an hour or more. The results showed that $40 \%$ of the barriers to participation in physical activity programs and $77.5 \%$ of the facilitators belonged to the intrapersonal domain. The most prevalent barrier was "current health condition" and the facilitator was the desire "to be healthier". Women perceived more barriers than men. The conclusion is that barriers and facilitators from the intrapersonal domain, related to health, are the main factors involved in users' engagement and retention in programs and interventions to promote physical activity, developed in basic healthcare services in the state of Pernambuco.

Exercise; Health Promotion; Primary Health Care

\section{Resumen}

En Brasil, la actividad física es el eje prioritario de las acciones de promoción de la salud en el Sistema Único de Salud (SUS). El presente estudio tiene como objetivo describir el perfil de los usuarios, barreras y facilitadores para la participación en programas de promoción de actividades físicas en la atención básica de la salud. Se trata de un estudio transversal con alcance estatal, realizado en los municipios de Pernambuco. Se entrevistaron de 2 a 21 individuos, por municipio, mediante un instrumento previamente testado y validado en las siguientes dimensiones: sociodemográfica; estado de salud; participación; información sobre el programa; motivos de participación; facilitadores para la práctica; barreras para la práctica $y$ preferencia de actividades. Se realizaron análisis descriptivos e inferenciales (chi-cuadrado). La muestra fue de 1.153 usuarios, un 35,9\% con una edad comprendida entre los 41-59 años; un 90,1\% residentes en áreas urbanas; un 58,2\% de los usuarios participaban en actividades desde hacía por lo menos un año. La frecuencia semanal de 3-4 dias fue de $44,9 \%, y$ un $71,1 \%$ practicaban actividades de una hora o más. Se observó que el $40 \%$ de las barreras para la participación en los programas de actividad física, y un 77,5\% de los facilitadores relatados, fueron de dominio intrapersonal. La barrera más prevalente fue "estado actual de salud" y el facilitador fue "tener un estado mejor de salud". Las mujeres perciben más barreras que los hombres. Se concluye que las barreras y los facilitares de dominio intrapersonal, relacionados con la salud, son los factores implicados en el mantenimiento e implicación de los usuarios en los programas e intervenciones para la promoción de la actividad fisica, desarrollados por la atención básica a la salud del estado de Pernambuco.

Ejercicio; Promoción de Salud; Atención

Primaria de Salud
Recebido em 30/Abr/2019

Versão final reapresentada em 18/Set/2019

Aprovado em 09/Out/2019 